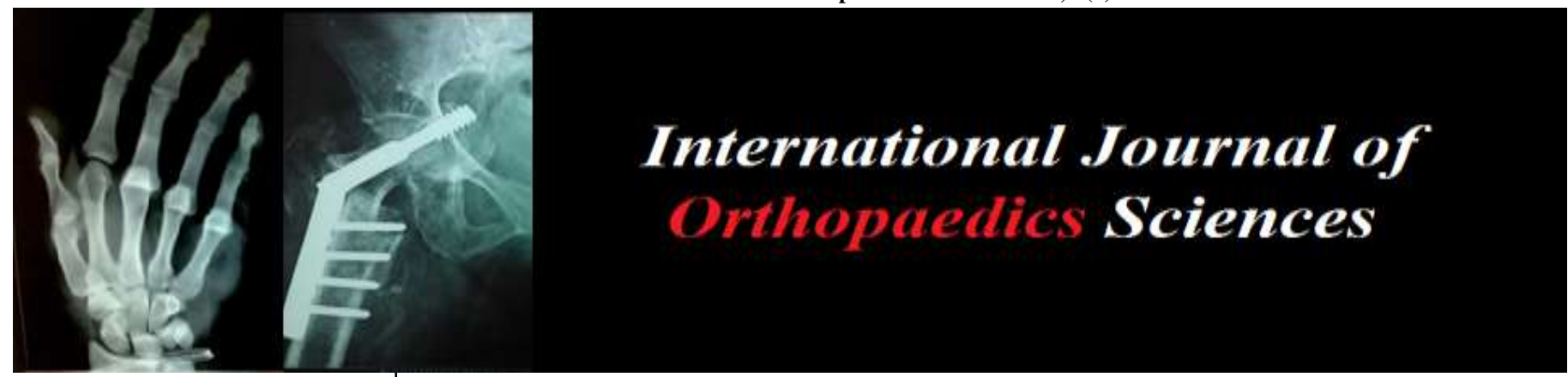

E-ISSN: 2395-1958

P-ISSN: 2706-6630

IJOS 2021; 7(2): 832-837

(C) 2021 IJOS

www.orthopaper.com

Received: 15-02-2021

Accepted: 21-03-2021

Dr. K Kanagasarathy

Department of Orthopaedics,

Meenakshi Hospital,

Thanjavur, Tamil Nadu, India
Corresponding Author: Dr. K Kanagasarathy

Department of Orthopaedics, Meenakshi Hospital,

Thanjavur, Tamil Nadu, India

\section{Paediatric diaphyseal forearm fractures: Is titanium elastic nailing a safe option?}

\section{Dr. K Kanagasarathy}

DOI: https://doi.org/10.22271/ortho.2021.v7.i2k.2710

\section{Abstract}

Paediatric forearm fractures are common injuries accounting for $40 \%$ of all the fractures in children. They are traditionally treated conservatively with casting. However there has been short and long term complications associated with such treatment and in recent times, closed reduction and flexible nailing has shown favourable clinical outcomes. In this prospective study, we evaluated functional outcome in closed forearm diaphyseal fractures of children and adolescents treated with titanium elastic intramedullary nails. All cases achieved bony union at an average of eight weeks and excellent functional outcome without significant complications. Nails were removed under anaesthesia after radiological union. Intramedullary nailing with elastic nails is an effective method of treatment for these unstable diaphyseal fractures of forearm in children and adolescents.

Keywords: Forearm fractures, children, titanium elastic nailing

\section{Introduction}

Injuries to the shaft of the radius and ulna are the most common reasons for children to receive orthopedic care. They include $40 \%$ of all the childhood fractures ${ }^{[1]}$. Frequency of radius and ulna are highest among in children aged 14 years or less. Common mechanism of injuries include falling on outstretched hands, direct trauma (ex: nightstick fracture), non-accidental injuries.

Biomechanical studies have suggested that diaphysis of forearm in children have an increased vulnerability to fracture and are highly unstable ${ }^{[2]}$. They are traditionally treated conservatively with casting. However there has been short and long term complications associated with such treatment and in recent times, closed reduction and flexible nailing has shown favourable clinical outcomes.

\section{Materials and Methods}

This was a prospective study conducted at Meenakshi Hospital, Thanjavur. Around 15 patients in the age group between 4 and 14 years with diaphyseal forearm fractures were treated with titanium elastic nails between August 2019 and August 2020 were included in the study. The distribution of the cases are as follows:



Age group

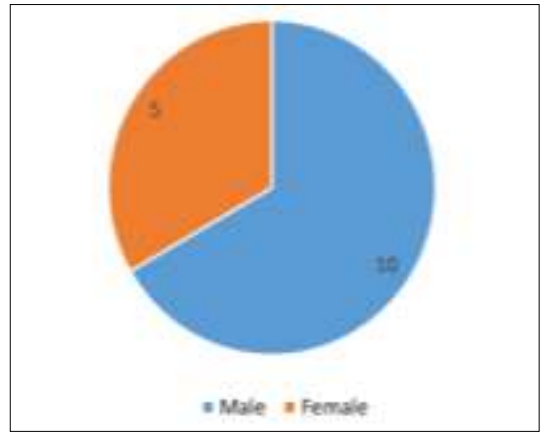

Sex distribution 


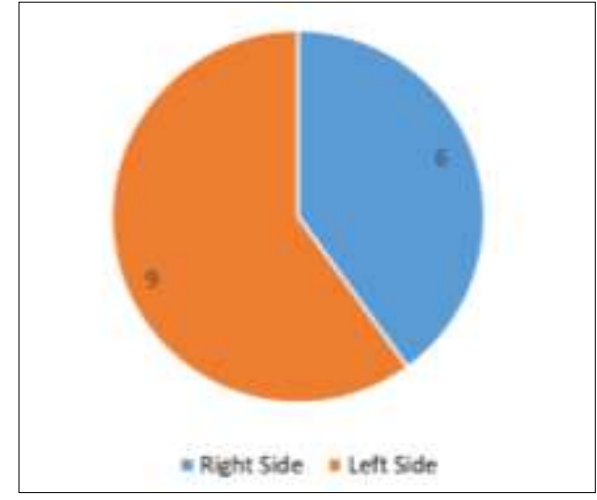

Side of fracture

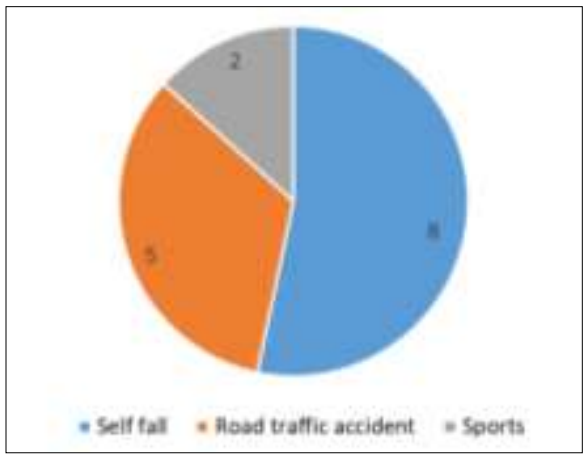

Mode of injury
After a proper evaluation with relevant history and clinical examination, standard anteroposterior and lateral radiographs of forearm were taken. They were operated by a single surgeon under general anaesthesia. Closed reduction under CArm guidance and internal fixation with titanium elastic nails was the common surgery performed for all the cases. They were discharged on the second postoperative day with a plaster. They were followed up periodically for clinical outcome and radiographs monthly for about 6-months. Fracture union was assessed radiologically by callus formation and disappearance of fracture lines and clinically by absence of pain. Range of movements of elbow, wrist and radio ulnar joints was assessed and compared with normal limb. Functional outcome was evaluated using Price et al. criteria.

\section{Operative technique}

All the children were operated under general anesthesia. (Fig.1). A pneumatic tourniquet is used most often, in case of need for open reduction arises. The instruments and implants are checked beforehand. (Fig.2.). generally, the less comminuted bone is fixed first, in case of comminution of the fractures. A small longitudinal skin incision was made on the lateral side of the distal metaphysis of the radius just above the physis after marking under $\mathrm{C}$-arm guidance. Entry point is made with awl in a direction first horizontally and then advanced obliquely (Fig.3).

Table 1: Price et al. grading criteria

\begin{tabular}{|c|c|c|c|}
\hline Outcome & Symptoms & Loss of forearm rotation & No. of patients \& percentage \\
\hline Excellent & No complaint with strenuous activity & $<150$ & $11(73.3 \%)$ \\
\hline Good & Mild complaint with strenuous activity & $150-300$ & $3(20.0 \%)$ \\
\hline Fair & Mild complaint with daily activities & $310-900$ & $1(6.66 \%)$ \\
\hline Poor & All other results & $>900$ & 0 \\
\hline
\end{tabular}

Titanium elastic nail with a proximal bend of 30 degrees with appropriate diameter is chosen and introduced into the distal end of the radius just above the physis radius and advanced with gentle hand movements to the reach the fracture site. The fracture is then manipulated and reduced under $\mathrm{C}$-arm guidance. (Fig.4). The nail is then advanced into proximal metaphysis until it lies at the level of radial neck.

Another small incision is made on dorsum of upper forearm just below the olecranon in order to avoid the physis. Entry hole is made with awl and suitable size nail is advanced in a similar manner across the fracture site distally up to the ulnar styloid. The diameter of both the nails in radius and ulna should be of the same diameter. The tip of the nails is bent and cut short and buried under the skin. Skin is closed with stapler or stitches.

Above elbow plaster with an arm pouch is given for 2 weeks' time till suture removal and active finger exercises encouraged. Suture removal done on $14^{\text {th }}$ postoperative day and mobilisation exercises started. They were followed up regularly with radiographs. Implant removal was done when there is radiological evidence of union, mostly under anaesthesia.

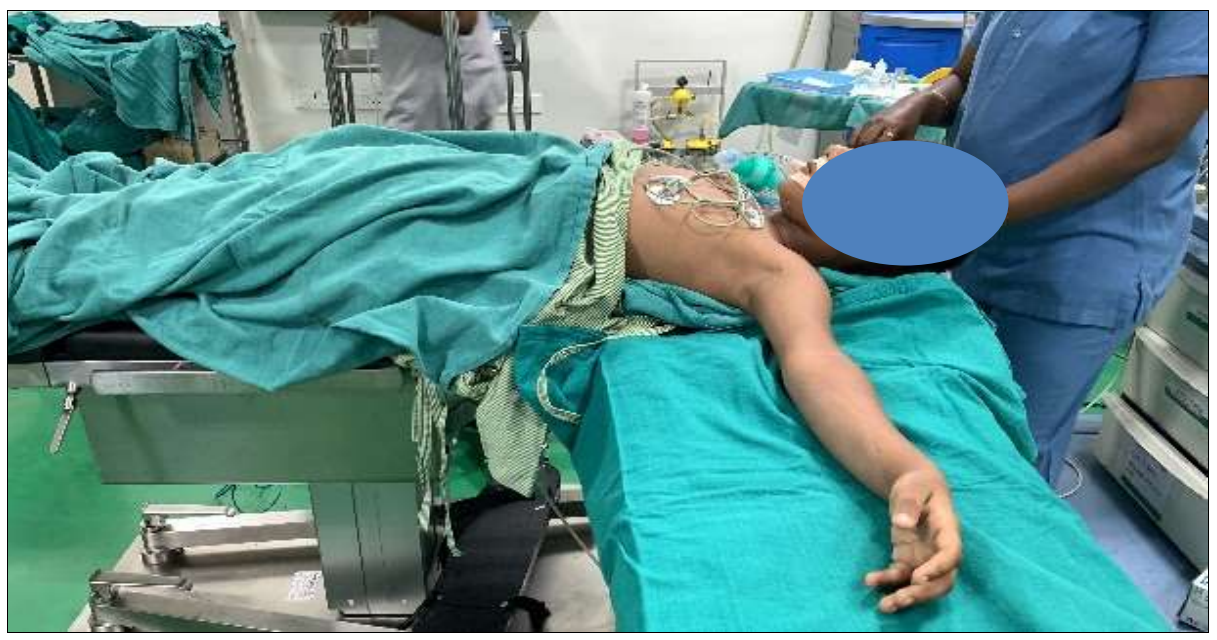

Fig 1: Positioning and anaesthesia 


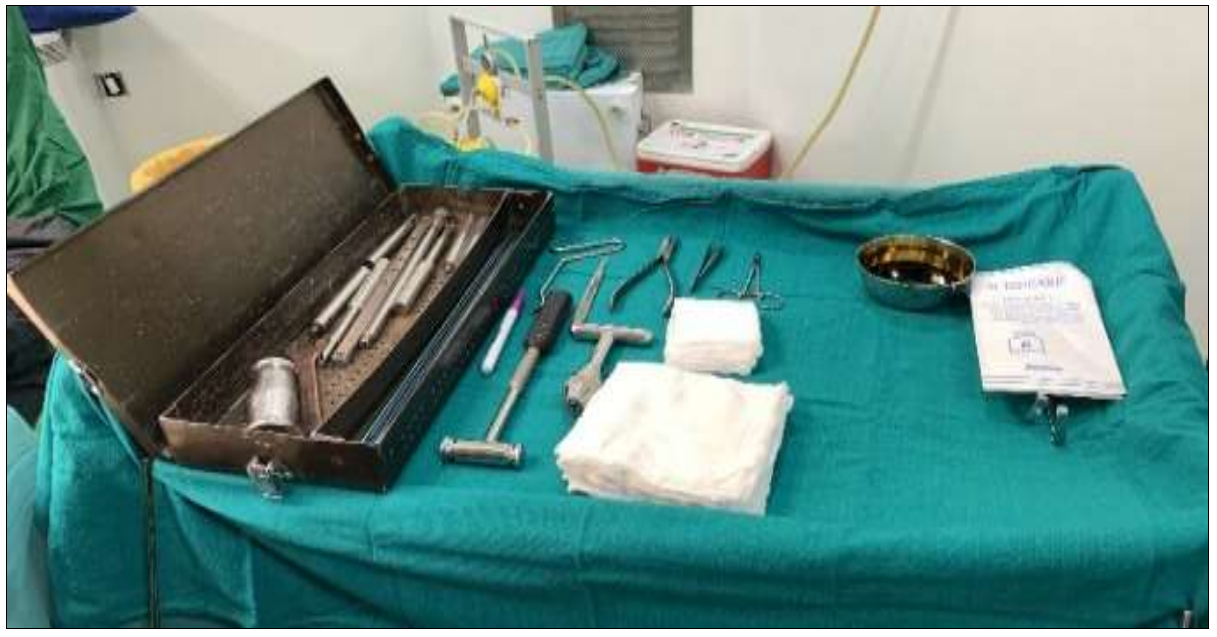

Fig 2: Instruments and implants

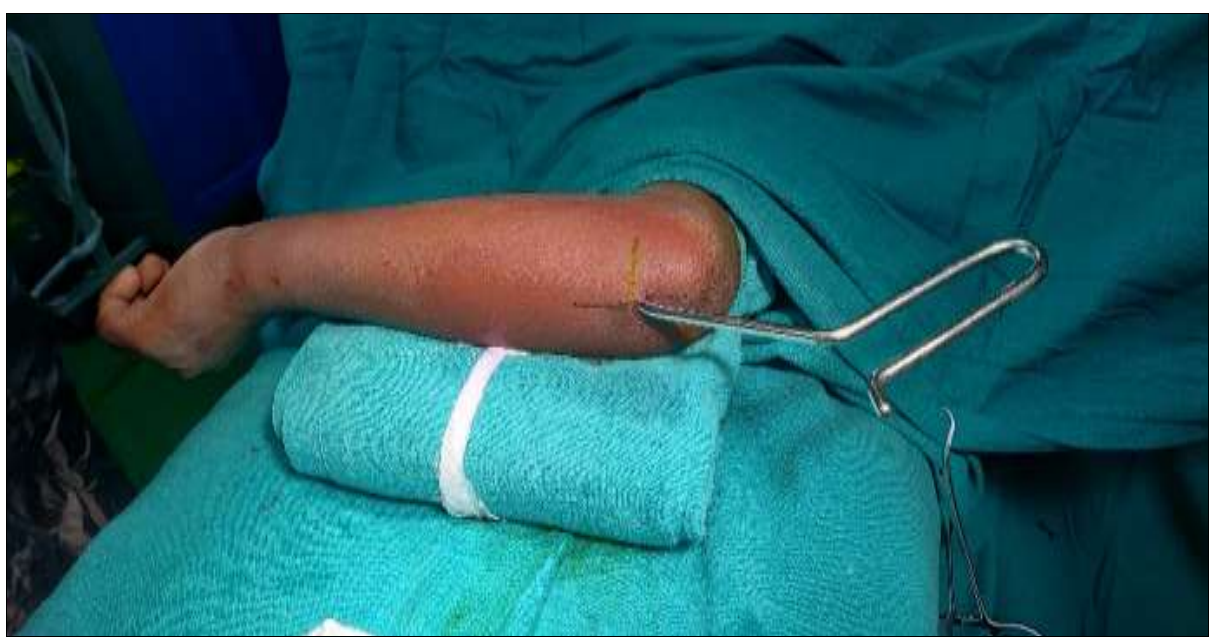

Fig 3: Entry site made with awl
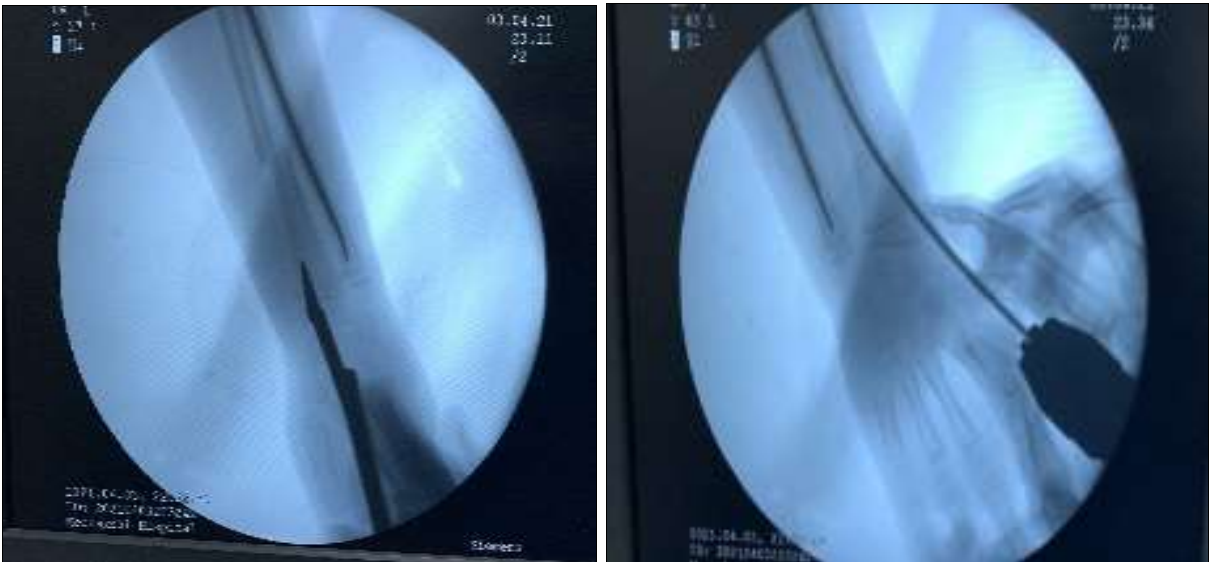

Fig 4: Intraoperative $\mathrm{C}$ arm pictures showing TENS nail insertion

\section{Results}

Serial radiographs at monthly intervals was performed to ascertain the fracture status. All cases achieved clinical and radiologic union at a mean of 2 months. There were no cases of non-union, mal-union, wound infection or refracture.
$12(73.3 \%)$ patients regained full range of motion, $2(20.0 \%)$ patients had mild restriction of movements and $1(6.66 \%)$ patient had moderate restriction of movements.

Functional scoring done according to Price et al. and there were 12 excellent, 2 good and 1 fair result. 


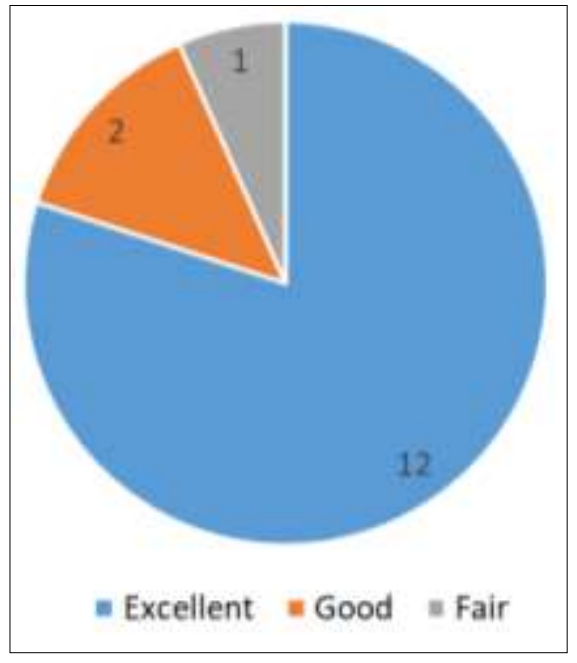

Functional outcomes

\section{Case-1:}

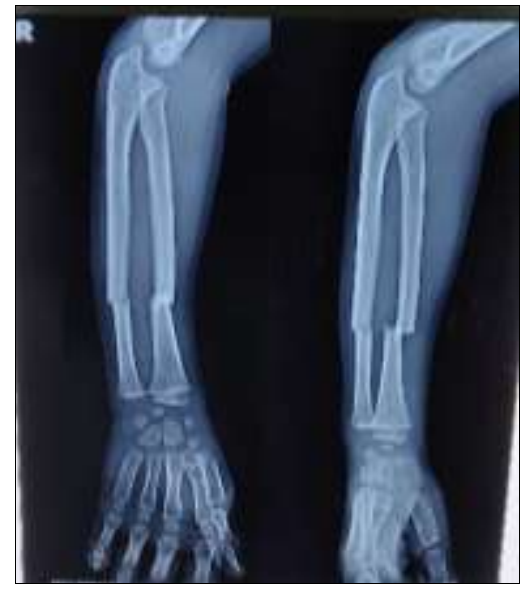

Pre-op X-ray

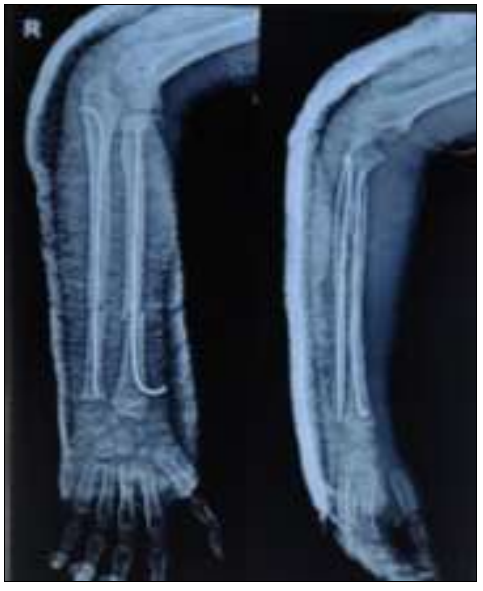

Immediate post-op

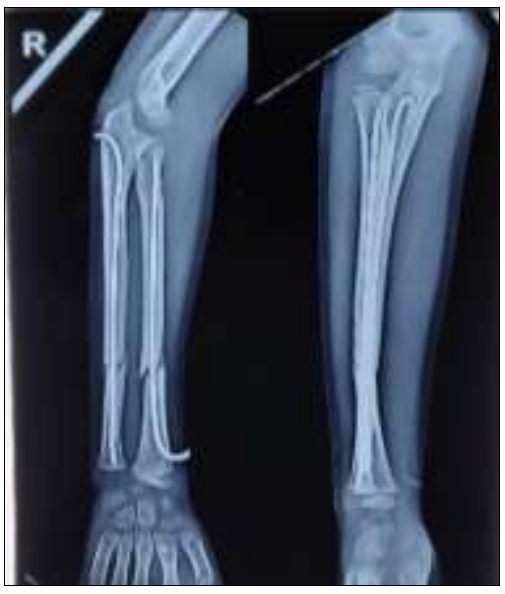

1 month post-op

Case 2:

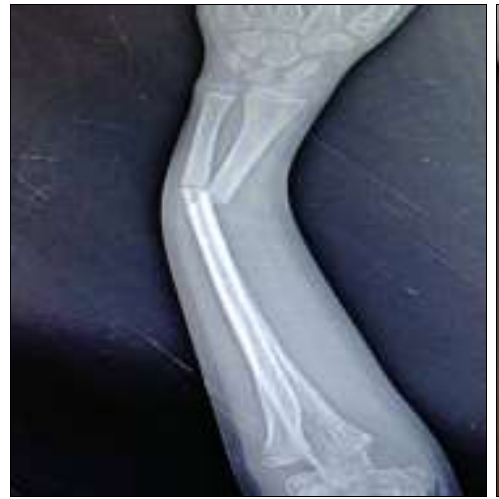

Pre-op X-ray AP view

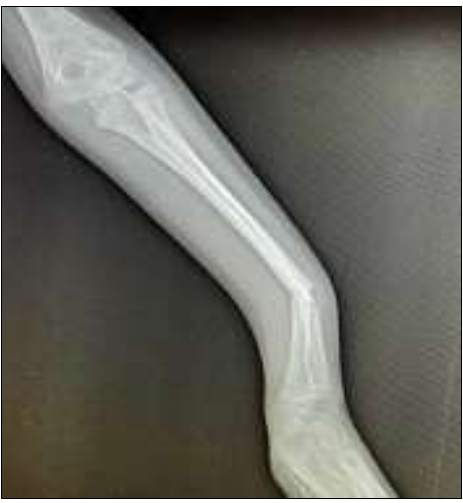

Pre-op X-ray lateral view 


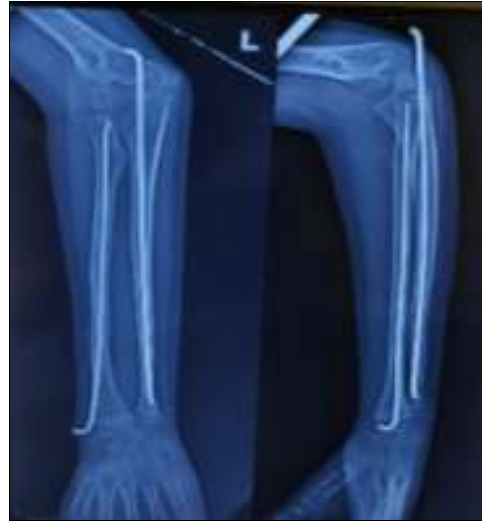

Immediate post-op X-ray

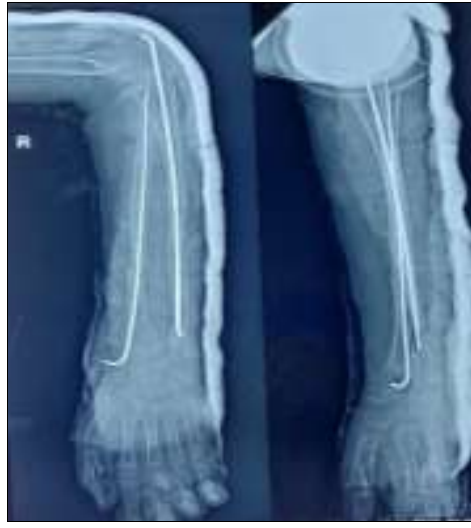

At 3 months follow-up

Case 3:

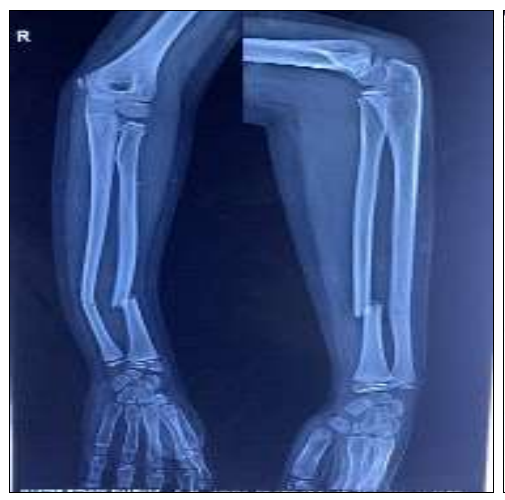

Pre-op X-ray AP \& lateral

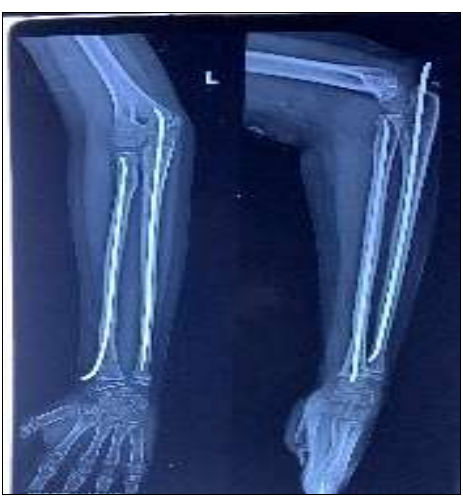

At 2 months follow-up
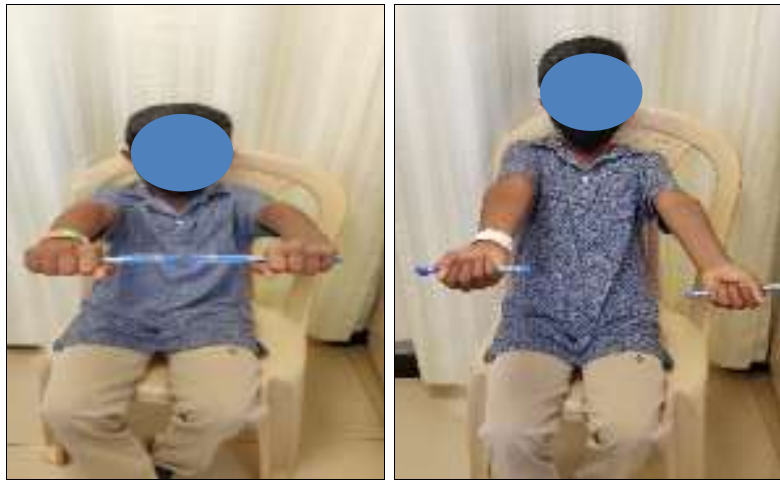


Followup range of movements

\section{Discussion}

Majority of diaphyseal fractures of forearm in children are being treated traditionally with casting. The major disadvantage with the conservative method is the unacceptable angulation and displacement that happens in the follow-up period, when the cast loosens, once the swelling subsides. Mal-union and resulting loss of function are the end results of such treatment. In such cases, surgical correction is warranted to restore anatomy and function.

In a study by Thomas et al. Kay et al. ${ }^{[3]}$ Eric N. Bowman et al. ${ }^{[4]}$ for those children who were treated conservatively, there was a significant loss of reduction in the range of $39 \%, 64 \%$ and $51 \%$ respectively especially in children above 10 years of age ${ }^{[12-14]}$. The major causes of failure include displacement of the fracture, angulation and mal-rotation. To add to the deformity issues, Rodriguez-Merchan ${ }^{[5]}$ in their literature stated that rotational deformity does not remodel at all.

Limb length discrepancy and loss of function including supination and pronation as a result of mal-union with angulation and rotation causing limitation in daily activities was described by Daruwalla et al. and Morrey et al. ${ }^{[6]}$.

Hence in order to restore anatomy and early mobilization to regain normal function, surgical management of these fractures is the procedure of choice. It also helps to avoid malunion and provide full functional range in pediatric both bone forearm fracture.

Most of these fractures can be reduced by closed means under IITV control, occasionally because of soft tissue interposition, open reduction may be needed. Range of movements encouraged as soon as pain tolerated and helps to avoid joint stiffness. Plaster slab is generally not required as internal fixation is adequate. However for initial few days, sling and a plaster is applied to reduce oedema in the postoperative period.

Among the many methods available for internal fixation, plates and screws were commonly used in adolescents and adults. The main advantage with titanium elastic nails is that it provides a stable three point fixation as well as rotational 
stability, micro-motion at fracture site, minimally invasive, preserves fracture hematoma and periosteal blood supply which promotes early fracture union.

In a retrospective study by Salonen A et al. ${ }^{[7]}$ on 75 children concluded that TENS nailing is considered a suitable treatment for all unstable forearm shaft fractures. In another study by Shah AS et al. ${ }^{[8]}$ sixty-one skeletally immature adolescents (mean age, 13.9 years; range, 11.5-16.9 years) were treated operatively for fracture both bones forearm and concluded that flexible nailing in adolescents was safe and effective in their series.

Alnaib $\mathrm{M}$ et al. ${ }^{[9]}$ also reported titanium elastic nailing in the pediatric age group is a minimally invasive procedure maintains alignment and promotes early union of fractures. The study by Furlan D et al. ${ }^{[10]}$ also suggests that flexible elastic nailing is the method of choice in children as it is minimally invasive and has good functional and cosmetic results.

\section{Conclusion}

Pediatric diaphyseal forearm fractures are unstable and challenging fractures. With the advent of titanium elastic nails, the treatment has been simplified and results are promising in terms of functional outcome with minimal or no complications. Hence it can be considered a better alternative to invasive procedures like plating.

\section{Conflicts of interest: None}

\section{Source of funding: Nil}

\section{References}

1. Jones K, Weiner DS. The management of forearm fractures in children: a plea for conservatism. J Pediatr Ortho 1999;19:811-5.

2. Sinikumpu JJ, Pokka T, Serlo W. The changing pattern of pediatric both-bone forearm shaft fractures among 86,000 children from 1997 to 2009. Eur J Pediatr Surg 2013;23:289-96.

3. Thomas EM, Tuson KW, Browne PS. Fractures of the radius and ulna in children Injury 1975;7(2):120-124.

4. Eric N, Bowman MPH, Charles Mehlman T, DO MPH, Christopher Lindsell J PhD, Junichi Tamai MD. Nonoperative treatment of both-bone forearm shaft fractures in children: Predictors of early radiographic failure, J Pediatr Orthop 2011;31(1):23-32.

5. Rodríguez-Merchán EC. Pediatric fractures of fore arm. Clin Orthop Relat Res 2005;432:65-672.

6. Daruwalla JS. A study of radioulnar movements following fractures of the forearm in children Clin Orthop 1979;139:114-120.

7. Salonen A, Salonen H, Pajulo O. A critical analysis of postoperative complications of antebrachium TENSnailing in 35 children, Scand J Surg 2012;101(3):216-21.

8. Shah AS, Lesniak BP, Wolter TD, Caird MS, Farley Vander FA, Have KL. Stabilization of adolescent bothbone forearm fractures: a comparison of intramedullary nailing versus open reduction and internal fixation, $\mathbf{J}$ Ortho Trauma 2010;24(7):440-7.

9. Mustafa Alnaib, Razvan Taranu, Sandesh Lakkol, Ehab Aldlyami, Ilhan Alcelik, Christopher Tulloch. Radiusonly intramedullary nailing for both-bones diaphyseal forearm fractures in children, Acta Ortho. Belg 2011;77:458-463.

10. Furlan D, Pogorelic Z, Biocic Juric, Budimir D, Todoric
$\mathrm{J}$, Mestrovic J et al. Elastic stable intramedullary nailing for paediatric long bone fractures: experience with 175 fractures, Scandinavian Journal of Surgery 2011;100(3):208-215. 